\title{
TRANSTORNO DO ESPECTRO AUTISTA NA ODONTOLOGIA
}

\author{
AUTISTIC SPECTRUM DISORDER IN DENTISTRY
}

Adyelle Dantas Ribeiro ${ }^{1}$

\begin{abstract}
RESUMO: Objetivo: Revisar a literatura acerca da importância das condições de saúde bucal do indivíduo com Transtorno do Espectro Autista (TEA). Método: Foram realizadas buscasbibliográficas nas bases de dados Science Direct, SciELO, PubMed e Google Acadêmico. As publicações indexadas nessas bases de dados foram identificadas por meio dos seguintes descritores: "Saúde Bucal", "Transtorno do Espectro Autista”, “ Assistência Odontológica para Pessoas com Deficiências ”, e dos respectivos termos em inglês: "Oral Health", "Autism Spectrum Disorder" e "Dental Care for Disabled". Inicialmente, os estudos foram selecionados apenas pelos títulos e resumos. Foram incluídos artigos científicos publicados entre 2011 e 2021 . Foram excluídas as teses, dissertações e estudosque não abordavam a temática principal da presente pesquisa. Resultados: Verificou-se que os pacientes com TEA apresentam algumas condições que favorecem o aparecimento de algumas doenças da cavidade oral, que podem estar associadas ao uso crônico de medicamentos, aos hábitos de alimentação e higiene, motivação dos cuidadores, cooperação do paciente e segurança profissional. Conclusão: É necessário enfatizar a importância do conhecimento sobre saúde bucal para os cuidadores, frequência de-consultas, manejo do paciente, acessibilidade aos serviços de saúde e capacitação profissional.
\end{abstract}

Palavras chave: Assistência Odontológica para Pessoas com Deficiências. Saúde Bucal. Transtorno do Espectro Autista.

ABSTRACT: Objective: To review the literature on the importance of oral health conditions for individuals with Autistic Spectrum Disorder (ASD). Method: Bibliographic searches were performed in the Science Direct, SciELO, PubMed and Google Scholar databases. The publications indexed in these databases were identified using the following descriptors: "Oral Health", "Autistic Spectrum Disorder", "Dental Assistance for People with Disabilities", and the respective terms in English:

\footnotetext{
${ }^{1}$ Doutoranda em Clínicas Odontológicas, Mestre em Saúde e Sociedade, Universidade Estadual da Paraíba UEPB, Campina Grande - PB.
} 
"Oral Health", "Autism Spectrum Disorder "and" Dental Care for Disabled ". Initially, studies were selected only by titles and abstracts. Scientific articles published between 2011 and 2021 were included. Theses, dissertations and studies that did not address the main theme of this research were excluded. Results: It was found that patients with ASD have some conditions that favor the appearance of some diseases of the oral cavity, which may be associated with chronic use of drugs, eating and hygiene habits, motivation of caregivers, patient cooperation and safety professional. Conclusion: It is necessary to emphasize the importance of knowledge about oral health for caregivers, frequency of consultations, patient management, accessibility to health services and professional training.

Keywords: Oral Health; Autism Spectrum Disorder; Dental Care for Disabled. 


\section{INTRODUÇÃO}

O Transtorno do Espectro do Autismo (TEA) trata-se de um grupo de deficiências de neurodesenvolvimento que comprometem a interação social, a comunicação e restrição ou repetição de estereótipos comportamentais (KHOLOOD, et al. 2020; JUMA, et al. 2019). Normalmente, a criança autista apresenta deficiência na comunicação verbale dificuldades de desenvolver interações sociais, compartilhar experiências e interesses, atraso ou falta total de desenvolvimento linguístico, bem como adesão inflexível aos rituais (ABDULMONEM, et al. 2020; EMAN, et al. 2020).

O TEA, de maneira geral, se apresenta entre os três primeiros três anos de vida e geralmente afeta mais homens do que mulheres em uma proporção entre $3 \mathrm{e}$ 4:1. A Organização Mundial da Saúde (OMS) estima uma prevalência global de TEA de 1:160 pessoas, com um aumento da prevalência nas últimas décadas (CRUZ, et al. 2017; EADES, et al. 2019).

Diante das dificuldades de interação e comunicação inerentes a própria condição do paciente, observa-se a falta de cooperação dos mesmos com o tratamento odontológico, por terem dificuldades em interagir com outras pessoas e em compreendere seguir instruções, o que acarreta prejuízos a saúde bucal desses pacientes, fato este observado em alguns estudos da literatura (KHANNA, et al. 2011; DU et al. 2019).

O atendimento odontológico para esses pacientes é negligenciado, pois eles muitas vezes enfrentam adversidades relevantes no acesso atendimento odontológico devido à dificuldade de localizar um dentista especializado no tratamento, conforme necessário (VAJAWAT, et al. 2012; STEIN et al., 2012). A saúde bucal é uma parte importante da saúde em geral. Problemas bucais podem causar dor, sofrimento e limitaçãofuncional que afetam a qualidade de vida e o bemestar do indivíduo.

Diante da relevância de se conhecer as principais alterações presentes na cavidade oral de pacientes com TEA, bem como as dificuldades encontradas pelos 
cirurgiões- dentistas no atendimento destes pacientes, e percebendo também uma carência de estudosque abordem esta temática, o presente artigo tem como objetivo realizar uma revisão de literatura sobre o atendimento odontológico ao paciente com Transtorno do Espectro Autista (TEA), abordando a condição de saúde bucal, conscientização dos cuidadores e capacitação profissional.

\section{MATERIAIS E MÉTODO}

Foi realizada uma revisão crítica da literatura por meio da busca bibliográfica nas bases de dados Science Direct, SciELO, PubMed e Google Acadêmico. As publicações indexadas nessas bases de dados foram identificadas por meio dos seguintes descritores: "Saúde Bucal", "Transtorno do Espectro Autista", " Assistência Odontológica para Pessoas com Deficiências ", e dos respectivos termos em inglês: "Oral Health", "AutismSpectrum Disorder" e " Dental Care for Disabled". Inicialmente, os estudos foram selecionados apenas pelos títulos e resumos. Foram incluídos artigos científicos publicados entre 2011 e 2021. Foram excluídas as teses, dissertações e estudos que não abordavam a temática principal da presente pesquisa.

\section{REVISÃO DE LITERATURA}

O Transtorno do Espectro do Autismo (TEA) foi descrito pela primeira vez pelo psiquiatra Léo Kanner, em 1943. Atualmente descrito pela American Psychiatric Association (2013) como um distúrbio do neurodesenvolvimento, que se manifesta em de uma forma séria ao longo da vida (NICOLAIDIS, et al. 2015; THOMAS et al. 2015). Trata-se de um transtorno do desenvolvimento que afeta a comunicação, o comportamento e a interação social, em que os indivíduos apresentam alguns sintomas que dificultam seu desempenho escolar, no trabalho e em várias áreas da 
sua vida, bem como interesses restritos e comportamentos repetitivos (MCKINNEY et al. 2014; DELLlet al. 2013).

O TEA é considerado um dos principais problemas de saúde pública na última década. Crianças autistas não conseguem desenvolver atenção conjunta, apresentam dificuldade de compartilhar informações usando uma linguagem falada, gestos e contatovisual, o que pode interferir no atendimento odontológico de forma negativa (BEATON, et al. 2013; ISONG et al. 2012).

De acordo com Alkahtani et al. (2014), para estabelecer um diagnóstico clínico confiável do TEA, é importante classificar os indivíduos de acordo com três níveis de gravidade desta condição, de acordo com os critérios a seguir:

\section{Nível 1 - "Requer suporte"}

Comunicação social: Na falta de estímulo apresenta déficits na comunicação social podendo causar perdas notáveis. Pode apresentar interesse reduzido em interagir socialmente.

Comportamento restrito e repetitivo: A inflexibilidade de comportamento causa interferência significativa na função em um ou mais contextos. Problemas com organização e o planejamento são obstáculos à independência.

\section{Nível 2 - "Requer suporte substancial”}

Comunicação social: Graves déficits na comunicação social verbal e não verbal, bem como de habilidades. Perdas sociais aparentes mesmo na presença de apoio.

Comportamento restrito e repetitivo: Inflexibilidade de comportamento, dificuldade em lidar com mudanças ou outros comportamentos restritivos / repetitivos. Sofrimento e / ou dificuldade de mudança de foco ou ações. 


\section{Nível 3 - "Requer suporte muito substancial"}

Comunicação social: Déficits graves nas habilidades de comunicação verbal e não verbal. Grande limitação em iniciar interações sociais e mínimo resposta às aberturassociais.

Comportamento restrito e repetitivo: Inflexibilidade de comportamento, extrema dificuldade em lidar com mudança ou outros comportamentos restritos / repetitivos. Grande sofrimento incluindo dificuldade para mudar de foco ou atitudes.

Com base nisso, indivíduos com necessidades especiais, incluindo o TEA, podem ter limitações consideráveis no desempenho da higiene oral devido às suas deficiências motoras, sensoriais e intelectuais, tornando necessária a interação entre os cuidadores e os profissionais de saúde bucal, devendo estes se capacitarem a fim de proporcionar condições dignas de saúde e qualidade de vida (NICOLAIDIS et al., 2014; RICHA et al.2014).

\section{TEA E CAVIDADE ORAL}

Pesquisas que investigaram o estado de saúde bucal de pacientes com TEA mostraram uma alta incidência de alterações na cavidade oral desses indivíduos. No quediz respeito a cárie, existe uma controvérsia, alguns estudos apresentam menor prevalência de cárie em pacientes com TEA e outros relatam uma taxa maior de cárie nesses pacientes (SHELDRICK, et al. 2012). Por outro lado, a má higiene oral e a doença periodontal foram altamente relatadas entre crianças com esta condição. Isso ocorre em decorrência da escovação dentária menos frequente quando comparado à crianças com desenvolvimento normal, associado ao uso de alguns medicamentos específicos para o TEA, que causam baixo fluxo salivar e aumentam as chances de desenvolvimento de problemas bucais. Foi relatado na literatura, 
também, alterações como: erupção dentária retardada, trauma e lesão, em decorrência do ato de morder os lábios, bruxismo, mastigação não nutritiva (ABDULMONEM, et al. 2020; EMAN, et al. 2020).

Além desses fatores diretos, os indivíduos com TEA também podem enfrentar outros desafios e barreiras que limitam o acesso aos cuidados de saúde bucal, como família de baixa renda e comportamento não cooperativo no atendimento odontológico, acentuando ainda mais as alterações na cavidade oral desses pacientes (KHANNA, et al. 2011; DU et al. 2019).

\section{ATENDIMENTO ODONTOLÓGICO}

Os cuidados dentários para crianças com necessidades especiais são frequentemente negligenciados tanto pelos dentistas como pelos pais, visto que os dentistas podem relutar em tratar crianças com necessidades especiais devido à insegurança e à falta de conhecimento de vários distúrbios que acometem pacientes comnecessidades especiais (KHOLOOD, et al. 2020; JUMA, et al. 2019). Além disso, os pais geralmente priorizam outras condições médicas à frente da saúde bucal, devido à falta de consciência da importância da odontologia. Outros fatores que podem prejudicar o acesso à saúde bucal incluem dificuldade na linguagem e barreiras psicossociais, estruturais e culturais (MCKINNEY et al. 2014; DELLI et al. 2013).

Resistência ao tratamento dentário, complicações relacionadas com a condição desaúde e dificuldade em encontrar um dentista competente foram citadas por tutores de crianças autistas (BEATON, et al. 2013; ISONG et al. 2012). Os pais relataram a incapacidade de conter as crianças durante o tratamento dentário, bem como na sala de espera de clínicas odontológicas. Portanto, o atendimento ao paciente com necessidade especial trata-se de um desafio para a equipe dentária, devido à capacidade de atenção inadequada e ao baixo limiar de frustração e habilidades de comunicação reduzidas, sendo frequentemente um processo 
demorado e que requer numerosas visitas para iniciar o tratamento de fato (SHELDRICK, et al. 2012).

A natureza invasiva do atendimento odontológico, hipersensibilidade de crianças com TEA, a estimulação sensorial (som, toque e luz) podem desencadear respostas violentas e indesejadas durante o tratamento dentário, devendo-se lançar mão de técnicas de manejo para facilitar esse processo (VAJAWAT, et al. 2012; STEIN et al., 2012).

De acordo com Cruz, et al. 2017, uma das principais técnicas conhecidas para modelagem de comportamento é o uso de recursos visuais, que são empregados para modificar o comportamento das crianças com TEA e ajuda na preparação para o tratamento dentário. Trata-se de uma técnica onde se usam uma série de imagens ou desenhos que tornam crianças com TEA familiarizadas com os instrumentos odontológicos e os protocolos de tratamento, fornecendo as informações em etapas, permitindo que processo de aprendizagem possa ocorrer (SHELDRICK, et al., 2012)..

Para Eades, et al. (2019), em razão do atendimento odontológico ser a principal necessidade de saúde negligenciada em crianças com necessidades especiais de saúde e deficiência, essas crianças apresentam maiores necessidades odontológicas em comparação com os indivíduos sem TEA, e em geral, são indivíduos mais propensos a ter pior saúde bucal do que indivíduos sem essas deficiências.

\section{IMPORTÂNCIA DO CUIDADOR}

Os cuidadores de pacientes com TEA têm um papel fundamental na saúde geral e bucal desses pacientes, sendo, em geral, os pais são considerados vitais para melhorar a saúde bucal dos seus filhos (MCKINNEY et al. 2014; DELLI et al. 2013).

Estudos de Kholood, et al. (2020) e Juma, et al.(2019), mostram que a grande maioria dos pais não têm nenhuma informação sobre práticas de higiene oral e a 
maioriaescovava os dentes dos seus filhos apenas uma vez por dia, e alguns destes nunca escovaram os dentes dos seus filhos.

Pais de crianças com TEA estão, muitas vezes, sobrecarregados e sujeitos a fardos físicos, financeiros e psicológicos, que fazem com a saúde bucal seja negligenciada. Essas crianças dependem exclusivamente dos cuidadores para todas as suas necessidadesdiárias, alimentares e higiene oral (CRUZ, et al. 2017; EADES, et al. 2019). Atitudes negativas dos pais em relação à saúde bucal foram associadas as piores condições de saúde bucal dos filhos, enquanto atitudes positivas são um forte preditor de saúde bucal de crianças com TEA (BEATON, et al. 2013; ISONG et al. 2012).

Diante disso, fica evidente a importância do cuidador para a manutenção, conjuntamente com o cirurgião dentista, da saúde bucal dos pacientes com TEA, sendo necessário intervir na conscientização dos cuidadores no sentido de melhorar as condições orais e na qualidade de vida como um todo.

\section{DISCUSSÃO}

A saúde bucal é parte integrante da saúde geral do paciente, em razão disto, problemas orais podem ser fonte de dor, sofrimento e deficiência funcional e pode afetara qualidade de vida e o bem-estar do indivíduo. Pacientes com TEA enfrentam desafios notáveis ao receber ajuda odontológica, já que algumas de suas condições de saúde bucal podem ser atribuídas a uso de medicamentos ou a prática de hábitos orais deletérios, que podem interferir também na auto estima e satisfação com a saúde bucal por parte desses indivíduos (VAJAWAT, et al. 2012; STEIN et al., 2012).

Sujeitos com TEA não têm características orais peculiares relacionadas à sua patologia, no entanto, seus distúrbios têm consequências na cavidade oral, de modo quea saúde bucal das pessoas com TEA é pior do que a população em geral (KHANNA, et al. 2011; DU et al. 2019). Visto que esses indivíduos têm uma pior colaboração emexercer a higiene oral adequada, pode-se esperar que o risco de cárie seja maior nesses pacientes devido às dificuldades em escovar e passar fio 
dental seus dentes, possivelmente em virtude da falta de habilidades manuais, resultando em higiene oral inadequada. Portanto, a literatura atual mostra a validade de se considerar TEA como um indicador dealto risco de cárie e higiene oral pode ser o indicador de risco mais influente associado a novas cáries em crianças com TEA (ABDULMONEM, et al. 2020; EMAN, et al. 2020). Entende-se que alguns fatores podem afetar a eficácia da prevenção e do tratamento, como falta de controle adequado, dificuldade durante o atendimento odontológico, desatenção à dor do paciente ou necessidades de tratamento, além de problemas de comunicação e mau comportamento (VAJAWAT, et al. 2012; STEIN et al., 2012). No entanto, é imprescindível estabelecer vínculo com estes pacientes para que permitam o seu cuidado, portanto, os profissionais de odontologia devem ser capacitados e dispostos a desenvolver estratégias para realizar cuidados que promovam a saúde bucal desses pacientes, sem gerar angústia para eles e seus familiares, devendo-se estabelecer uma relação baseada na confiança entre o profissional, paciente e familiares (MCKINNEY et al. 2014; DELLI et al. 2013).

\section{CONCLUSÕES}

Foi apresentado o papel do odontólogo na esfera do indivíduo com Transtorno do Espectro Autista, bem como as principais particularidades desses indivíduos, no que diz respeito às condições bucais e sociais dos acometidos. Além disso, destacou-se a importância do conhecimento sobre saúde bucal pelos cuidadores, frequência de consultas, acessibilidade de serviços de saúde e capacitação profissional. 


\section{REFERÊNCIAS BIBLIOGRÁFICAS}

KHOLOOD, A.S.A.; ALDHALAAN, M.H.; MONEER, Z.; MOHAMMED, A.; AMAN, J.; REEM, M.A.; ABDULAZIZ, M.A.; KIRANK, G.; HEZEKIAH, M; Challenges of Autism Spectrum Disorders Families Towards Oral Health Care inKingdom of Saudi Arabia; Pesqui Bras Odontopediatria Clin Integr. 20(1): 1-7; 2020.

JUMA, O.S.A.; ESHRAQ, Z.E.; ABDULWAHA, M.A.Z.; AESA, A.J; Oral Health Status and Treatment Needs for Children with Special Needs: A Cross- Sectional Study; Pesqui Bras Odontopediatria Clin Integr. 19(1):1-10; 2019.

ABDULMONEM, A.A.; MANSOUR, H. A.A.; MOHAMMED, S.A.; Brief report: At-home oral care experiences and challenges among children withAutism Spectrum Disorder; Res Autism Spectr Disord. 79 (1): 1-10; 2020.

EMAN A.E.A.; Autism And Pediatric Dentistry: A Literature Review; Egypt Dent J. 66(1): 789798; 2020.

CRUZ, V.S.A.; CRUZ, T.A.A.A.; BANDEIRA, M.A.S.; GOMES, D.D.R.;SILVA, L.T.C.; SANTOS, V.C.B.; Rev Bras Odontol. 74(4):294-299; 2017.

EADES, D.; LEUNG,P.; CRONIN,A.; MONTEIRO, J.; JOHNSON, A.;REMINGTON, A; UK dental professionals' knowledge, experience and confidence when treating patients on the autism spectrum; Br Dent J. 227(6):505-510; 2019.

KHANNA, R.; MADHAVAN, S.S.; SMITH, M.J.; PATRICK, J.H.; TWOREK, C.; BECKERCOTTRILL B. Assessment of health-related quality of life among primary caregivers of children with autism spectrum disorders. J Autism Dev Disord. 41(1):1214-27; 2011.

DU, R.Y.; YIU, C.K.; KING, N.M; Oral health behaviours of preschool children with autism spectrum disorders and their barriers to dental care. J Autism Dev Disord. 49:453-59; 2019.

VAJAWAT, M.; DEEPIKA, P. C; Comparative evaluation of oral hygiene practices and oral health status in autistic and normal individualsJ Int Soc Prev Community Dent, 2, 58-63, 2012.

STEIN, L.; POLIDO, J.; NAJERA, S.; CERMAK, S. Oral Care Experiences and Challenges in Children, with Autism Spectrum Disorders. Paediatr Dent, 34: 387-391; 2012.

NICOLAIDIS, C.; RAYMAKER, D.; ASHKENAZY E et al. "Respect the way I need to communicate with you": Healthcare experiences of adults on the autismspectrum. Autism. 19(1): 824-831; 2015.

THOMAS, N.; BLAKE, S.; MORRIS, C.; MOLES, D. Autism and primary caredentistry: parents' experiences of taking children with autism or working diagnosis of autism for dental examinations. Int J Paediatr Dent, 28: 226-238;2015.

MCKINNEY, C.; NELSON, T.; SCOTT, J.; HEATON, L.; VAUGHN.; LEWIS Predictors of unmet dental need in children with autism spectrum disorder: results from a national sample. Acad Paediatr; 14: 624-631; 2014.

DELLI, K.; REICHART, P.; BORNSTEIN, M.; LIVAS, C; Management of children with autism spectrum disorder in the dental setting: Concerns, behavioural approaches and recommendations. Med Oral Patol Oral Cir Bucal;e862-e868.; 2013. 
BEATON, L.; FREEMAN, R.; HUMPHRIS, G. Why are people afraid of the dentist? Observations and explanations. Med Princ Pract; 23: 295-301, 2013.

ISONG, I.; RAO, S.; HOLIFIELD, C ET AL. Addressing dental fear in children with autism spectrum disorders. Clin Paediatr; 53: 230-237, 2014.

ALKAHTANI, Z.; STARK, P.; LOO, C.; WRIGHT, W.; MORGAN, J.; SAUDI, U.S; Dental student attitudes toward treating individuals with developmental disabilities. J Dent Educ; 78: 1145-115, 2014.

NICOLAIDIS, C.; KRIPKE, C.; RAYMAKER, D; Primary care for adults on the autism spectrum. Med Clin North Am; 98: 1169-1191, 2014.

RICHA, Y.R.; PURANIK, M.P; Oral health status and parental perception of child oral health related quality-of-life of children with autism in Bangalore, India. J Indian Soc Pedod Prev Dent; 32:135-139, 2014.

SHELDRICK, R.C.; NEGER, E.N.; SHIPMAN, D.; PERRIN, E.C; Quality of life of adolescents with autism spectrum disorders: concordance among adolescents' self-reports, parents' reports, and parents' proxy reports. Qual LifeRes. 21:53-57, 2012. 